\title{
The Capacity of Average and Peak Power Constrained Fading Channels with Channel Side Information
}

\author{
Mohammad Ali Khojastepour and Behnaam Aazhang \\ Department of Electrical and Computer Engineering, Rice University \\ 6100 Main St., MS-366, Houston, TX 77005 \\ Email: $\{$ amir, aaz\}erice.edu
}

\begin{abstract}
We derive the ergodic capacity of discrete-time fading channel with additive Gaussian noise subject to both peak and average power constraint. The average power can be interpreted as the cost that we incur to achieve a certain rate. On the other hand, the motivation of this analysis comes from the fact that there is also a peak power limitation in practical communication system. It is been shown that the optimal power adaption is no longer water-filling or constant power adaption which is the case where there is no limitation on the peak power. The numerical results show that the importance of peak power constraint become negligible for relatively low available average power, while it is limiting the capacity to be finite even as available average power goes to infinity.
\end{abstract}

\section{INTRODUCTION}

The Capacity of the various single-user memoryless channels with different constraints on the channel input and channel characteristics has been extensively studied since the early age of information theory [1], [2], [3], [4]. When the cardinality of the input alphabet is not finite, a constraint on the input is needed in order to have finite channel capacity. The most commonly used constraint is one on average power, for which the associated capacity of Gaussian channel is derived by Shannon [1]. Also, the capacity of the fading channel subject to average power constraint with perfect side information about the fading state both at the transmitter and the receiver is determined in [4] and the corresponding capacity without side information at the transmitter is found in [5]. However, there has not been much attention to the capacity of the channels subject to both average and peak power constraints, although its importance has been known for decades [1], and it better embraces the practical limitation of communication systems [6], [7], [8].

The Gaussian channel subject to an input peak power constraint is the first channel for which a constraint other than the average power is considered. The behavior of the Gaussian channels subject to peak and average power constraint is first studied in the original work of Shannon [1] for asymptotically low and high SNR in the context of bandlimited continuoustime Gaussian channel. The capacity of the Gaussian channel subject to both peak and average power constraint is later studied by Smith [2], and he showed that the capacity achieving distribution is discrete. The generalization of this result for the quadrature additive Gaussian channel, along with lower and upper bounds on the capacity of this channel can also be found in [3].
In this paper, we consider the capacity of the fading channel subject to the both peak and average power constraints under the assumption of the perfect side information about the fading state both at the transmitter and the receiver. We show that multiplexed Gaussian codebook with the rate and power adaption based on the fading states is sufficient to achieve the capacity. We prove a coding scheme which is more general to some extent, and subsumes the achievability of the rate for the specific case of peak and average power constraint over the fading channel. The converse part of the proof is derived from a newly derived cut-set theorem for the multi-state network [9], [10]. It is shown that as the peak power constraint approaches the average power, the capacity of fading channel under both peak and average power constraint approaches the capacity without side information at the receiver. On the other hand, as peak power goes to infinity, i.e. the peak power constraint being relaxed, the optimal power adaption becomes pure water-filling and the corresponding capacity approaches to the capacity of fading channel with perfect side information at the transmitter and the receiver.

The rest of the paper is organized as follows: We define some terminologies and notations, and also formulate the problem in Section II. Our main results which is the capacity of the fading channel with both peak and average power constraint is presented in Section III. The coding theorem and proof of the achievability is given in Section IV, and the converse is derived in Section V. In Section VI, we present the power adaption technique and the capacity results for the Rayleigh fading channel. Finally, we conclude in Section VII.

\section{Problem Formulation AND Notations}

We consider a discrete-time fading channel where in each time index $i$ the channel is characterized by two time-varying parameters, the channel gain $\sqrt{g[i]}$ and the additive Gaussian noise $z[i]$. The channel power gain $g[i]$ is also known as fading state of the channel. The random processes $g[i]$ and $z[i]$ are assumed to be ergodic and both independent from each other and the channel input. Let $\mathcal{N}_{0}$ denote the noise density and $B$ denote the received signal bandwidth. The additive noises $\{z[i]\}_{i=1}^{\infty}$ are assumed to be zero mean i.i.d. Gaussian random variables with the distribution $N\left(0, \mathcal{N}_{0} B\right)$. The channel power gains $\{g[i]\}_{i=1}^{\infty}$ are also assumed to be i.i.d. and non-negative real random variables with a known distribution such that the expected value of the channel power gain is unity. If $P_{i n}[i]$ denotes the input power at time $i$ and $\bar{P}$ denotes the average 


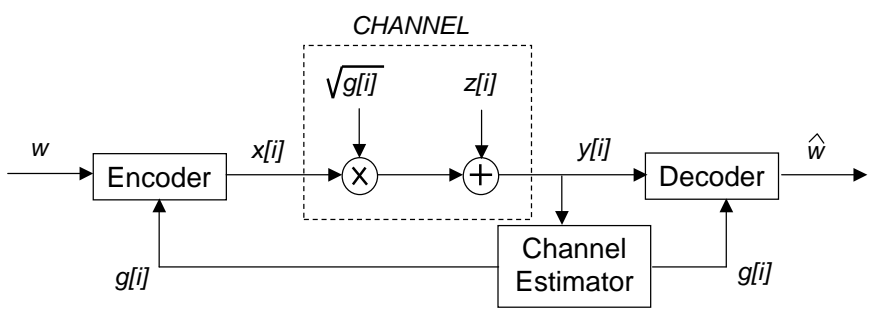

Fig. 1. System model

input power, then the instantaneous received signal-to-noise ratio without power control is given by $\gamma[i]=\bar{P} g[i] /\left(\mathcal{N}_{0} B\right)$, and its expected value is equal to $\bar{P} /\left(\mathcal{N}_{0} B\right) .{ }^{1}$ Since the distribution of the channel gain is assumed to be known, the distribution of $\gamma$ is known and will be denoted by $f(\gamma)$.

The channel model is illustrated in Figure 1. The message $w$ is encoded into the codeword $x$ of length $n$, which is transmitted over the time-varying channel as $x[i]$ at time $i$. The channel gain $g[i]$ varies from time to time and the coding scheme, which depends on both the message $w$ and the codeword length $n$, achieves the capacity of the channel if it is long enough to see all possible realizations of the channel. This will happen by letting the codeword length $n \rightarrow \infty$ due to the assumption of ergodicity. We assume that the instantaneous value of the channel gain $g[i]$ is known to the receiver at each time $i$, through a perfect channel estimator. Therefore the receiver can keep track of the channel state sequence $\{g[i]\}_{i=1}^{\infty}$ and it always has perfect channel state information. If a perfect and delay-less feedback channel is available then the transmitter also has the perfect channel state information. Thus, we consider the case that receiver has always perfect CSI but distinguish between the case that transmitter has perfect CSI or no CSI.

For any channel with continuous input alphabet, the capacity of the channel is infinite unless a constraint on the input power is considered. Usually, this constraint is in the form of an upper bound on the average input power $E_{\gamma}[P(\gamma)] \leq \bar{P}$, which is an indication of the cost that we incur to achieve a given rate. Here, $P(\gamma)$ is the power adaption based on the state of the channel, $\gamma$. Therefore, the instantaneous power adaption at time $i$ is given by $P_{i n}[i]=P(\gamma[i])$. However, in many practical systems, there is a limitation on the peak power [6], [7] due to the non-linear function of the amplifiers, and also for compliance with other standards (e.g. to limit the interference on the other communications). In this paper, we consider the case that both of the above mentioned constraints are present as

$$
\begin{gathered}
E_{\gamma}[P(\gamma)] \leq \bar{P} \\
\forall \gamma: P(\gamma) \stackrel{\text { a.s. }}{\leq} P_{\max }
\end{gathered}
$$

\footnotetext{
${ }^{1}$ Remark: Throughout the paper we shall refer to $\gamma$ as "Received SNR" for short, but we imply "Received SNR without power control" which only shows the variation of the fading state but not the power adaption. Clearly, with power control the actual value of the received SNR depends on the both the fading state and average power which is used in this state.
}

\section{CAPACITY ANALYSis}

The ergodic capacity of the fading channels with average power constraint is studied in [5], [4]. If the channel state information is not available at the transmitter, the capacity of the channel can be achieved by Gaussian codebook with constant power $\bar{P}$ and is given by average capacity formula [5] over all possible channel states

$$
C_{R C S I}(\bar{P})=E_{\gamma}[B \log (1+\gamma)] .
$$

It is also possible to interpret this average capacity formula by using the result of [11] on the capacity of the channels with side information at the receiver only. In fact, if the same input distribution maximizes the mutual information for all the channel states and also the sequence of the channel states are i.i.d. then it has been shown that the capacity is given by average capacity formula over all channel states [11].

On the other hand, if the perfect channel state information is available both at the transmitter and at the receiver, then capacity is again given by an average capacity formula with variable power adaption over all channel states [4]

$$
C_{T \& R C S I}(\bar{P})=E_{\gamma}\left[B \log \left(1+\frac{P(\gamma) \gamma}{\bar{P}}\right)\right],
$$

where power adaption over the channel states, $P(\gamma)$, is in the form of the water-filling solution given by

$$
\frac{P(\gamma)}{\bar{P}}=\left\{\begin{array}{ll}
\frac{1}{\gamma_{0}}-\frac{1}{\gamma}, & \gamma \geq \gamma_{0} \\
0, & \gamma<\gamma_{0}
\end{array},\right.
$$

such that it satisfies the average power constraint

$$
E_{\gamma}[P(\gamma)]=\bar{P}
$$

If the input signal is subject to a peak power constraint of the form (2) in addition to the average power constraint of (1), then we show that still a multiplexed Gaussian codebook with variable power would achieve the capacity of this channel. We derive an achievable rate by proving the existence of a coding scheme which satisfies the above constraints, and by the proof of the converse we show that it is the capacity of the channel. We also derive explicitly the form of the optimal power adaption which is needed to achieve the capacity in this case. It will be shown that the power adaption is divided into three regions based on the channel state. In the first region, we don't use the channel for the values of $\gamma<\gamma_{0}$. In other words, for the values of $\gamma$ less than a threshold $\gamma_{0}$ the input power is set to zero. In the second region, defined as the values of $\gamma_{0} \leq \gamma<\gamma_{1}$, the power adaption is in the form of water-filing. Finally, for the third region, which corresponds to $\gamma_{1} \leq \gamma$, a constant power equal to $P_{\max }$ will be used. The threshold values of the received SNR, $\gamma_{0}$ and $\gamma_{1}$ are determined such that the average power constraint is satisfied. It will be shown that for the fixed average power constraint by relaxing the peak-power constraint, the optimal power solution is in the form of the pure water-filling, which coincides with the results of [4]. On the other hand if the peakpower constraint remains unchanged, by relaxing the average power constraint the optimal power constraint is in the form of constant power adaption. In fact, in this case there would 
be no power adaption and capacity coincides with the capacity of the channel without side information at the transmitter. Specifically, we have the following theorem.

Theorem 1: The capacity of the fading channel with perfect channel state information both at the transmitter and the receiver, and the input signals subject to both peak and average power constraints of (1) and (2), respectively, is given by

$$
C_{T \& R C S I}\left(\bar{P}, P_{\max }\right)=E_{\gamma}\left[B \log \left(1+\frac{P(\gamma) \gamma}{\bar{P}}\right)\right],
$$

where the power adaption over the channel states, $P(\gamma)$, is in the form of

$$
P(\gamma)= \begin{cases}0, & \gamma<\gamma_{0} \\ \frac{\bar{P}}{\gamma_{0}}-\frac{\bar{P}}{\gamma}, & \gamma_{0} \leq \gamma<\gamma_{1} \\ P_{\max }, & \gamma_{1} \leq \gamma\end{cases}
$$

such that the threshold values $\gamma_{0}$ and $\gamma_{1}$ are determined from the following equations

$$
\begin{gathered}
\frac{\bar{P}}{\gamma_{0}}-\frac{\bar{P}}{\gamma_{1}}=P_{\max }, \\
E_{\gamma}[P(\gamma)]=\bar{P} .
\end{gathered}
$$

In order to prove the above theorem, it is necessary to show both the achievability of the rate $C_{T \& R C S I}\left(\bar{P}, P_{\max }\right)$ in (7) and the converse for the coding theorem for any coding schemes which satisfy both of the average and peak power constraints of (1) and (2), respectively. In Section IV, the achievability is proved for a broader class of power allocation schemes defined in Section IV that easily subsumes the class of $\mathcal{F}_{1}$ defined as

$$
\mathcal{F}_{1}=\left\{P(\gamma): E_{\gamma}[P(\gamma)] \leq \bar{P}, \quad \forall \gamma: P(\gamma) \stackrel{a . s .}{\leq} P_{\max }\right\}
$$

which is the class of power allocation schemes that satisfies both of the average and peak power constraint of (1) and (2). The converse part is proved based on the newly derived max-flow min-cut theorem for multi-state networks [9], [10] and it would be presented in Section V where we prove that achievable rate of any possible coding schemes for which the power control belongs to the class of $\mathcal{F}_{1}$ is not greater than the rate given by Theorem 1 .

\section{CODING THEOREM}

Consider a power allocation policy $P(\gamma)$ (policy in general might be stochastic or deterministic), which allocates the power $P(\gamma)$ for the received SNR value of $\gamma$. We define a class of power allocation policies as a given subset of all possible power allocation policies. A class of power allocation policies $\mathcal{P}$ may be defined as all power allocation policies that satisfy a given set of conditions which makes them distinguishable from other possible policies. For example $\mathcal{F}_{1}$ in (11) identifies the class of power allocation policies for which the power constraints of (1) and (2) are fulfilled.

The following theorem shows a coding scheme which gives an achievable rate for the power control policy which belongs to a given class. Therefore, supremum of the achievable rates in Theorem 2 over all possible power allocation policies of the same class is also achievable. Later, instead of taking the supremum which is very hard, we will pick a given policy in the class of $\mathcal{F}_{1}$ and show that this is actually the supremum of the achievable rate in this class by establishing the proof of the converse.

Theorem 2: Let $\mathcal{P}$ denote the class of power adaption policies defined as

$$
\mathcal{P}=\left\{P(\gamma): E_{\gamma}\left[B \log \left(1+\frac{P(\gamma) \gamma}{\bar{P}}\right)\right]<\infty\right\} .
$$

Consider a power control policy $P(\gamma) \in \mathcal{P}$, and let

$$
\mathcal{D}(P)=\{\gamma: P(\gamma) \neq 0\}
$$

denote the domain of $P(\gamma)$. There exists a coding scheme which achieves the rate $R$ defined as

$$
\begin{aligned}
R & =\int_{\mathcal{D}(P)} B \log \left(1+\frac{P(\gamma) \gamma}{\bar{P}}\right) f(\gamma) d \gamma \\
& =E_{\gamma}\left[B \log \left(1+\frac{P(\gamma) \gamma}{\bar{P}}\right)\right],
\end{aligned}
$$

with vanishing probability of error as the codeword length becomes infinitely large over the fading channel with perfect side information both at the transmitter and the receiver.

Proof: Clearly the values of the received SNRs, $\gamma$, are $0 \leq$ $\gamma<\infty$. Let $\rho=\inf _{\gamma \in \mathcal{D}(P)} \gamma$ and define the set

$$
\mathcal{D}_{1}=\{\gamma: \rho \leq \gamma \leq \rho+M\}
$$

for some integer value $\mathrm{M}$ (to be defined later). For the sake of the simplicity we assume that the power adaption policy $P(\gamma)$ is a deterministic policy and continuous over $\gamma$. Extension to general stochastic policies with possible discontinuity is not hard but not needed for deriving our results in this paper. In other words, we will later choose a deterministic and continuous policy which achieves the capacity of the channel over the class of $\mathcal{F}_{1}$.

Let $m$ be a positive integer and define $\gamma_{i}=\rho+\frac{i}{m}$ for all $i=0,1, \ldots, m M$. Also, let

$$
\xi_{i}=\int_{\gamma_{i}}^{\gamma_{i+1}} P(\gamma) d \gamma
$$

denotes the average power for any interval $I_{i}=\left[\gamma_{i}, \gamma_{i+1}\right)$.

Over a given time interval $[0, n]$, let $N_{i}$ denote the number of times that the received SNR is in interval $I_{i}$. By the stationarity and ergodicity of the channel gains $g[i]$ we have

$$
\frac{N_{i}}{n} \rightarrow \int_{\gamma_{i}}^{\gamma_{i+1}} f(\gamma) d \gamma
$$

For a given $n$, let

$$
n_{i}=\left\lfloor n \int_{\gamma_{i}}^{\gamma_{i+1}} f(\gamma) d \gamma\right\rfloor
$$

which is equal to $N_{i}$ for sufficiently large n. For any $i=$ $0,1, \ldots, m M$ define the rate $R_{i}$ as

$$
R_{i}=B \log \left(1+\frac{\xi_{i} \gamma_{i}}{\bar{P}}\right)
$$


and the overall rate $R^{(n)}$ as

$$
R^{(n)}=\sum_{i=0}^{m M} \frac{n_{i} R_{i}}{n}
$$

Clearly the rate $R_{i}$ defined in (19) is achievable over an AWGN channel with the received SNR $\frac{\xi_{i} \gamma_{i}}{\bar{P}}$. Therefore, there exist a code $\left(n_{i}, 2^{n_{i} R_{i}}\right)$ which encodes $2^{n_{i} R_{i}}$ equally likely messages, say $w_{i}$, with average input power $\xi_{i}$ and error probability $\epsilon_{n, i} \rightarrow 0$ as the codeword length $n_{i} \rightarrow \infty$.

Now we define the structure of the codebook and corresponding coding scheme. For a given $n$, the message index $w \in\left\{1,2, \ldots, 2^{n R^{(n)}}\right\}$ is mapped to the set of independent messages $\left\{w_{i}\right\}_{i=1}^{m M}$ by partitioning $n R^{(n)}$ bits into sets of $n_{i} R_{i}$ bits. Definition of the $n R^{(n)}$ in (20) ensures the existence of such a partitioning. The codebook for the channel consists of $m M$ independent gaussian codebook each with input average power $\xi_{i}$ and rate $R_{i}$ and code length $n_{i}$ for $i=1,2, \ldots, m M$. In order to transmit the message index $w$, we use the multiplexing strategy which transmit the corresponding message index $w_{i}$ defined in the aforementioned mapping over the channel when the received SNR $\gamma$ is in the interval $I_{i}$. By definition, $N_{i}$ denotes the number of time that the received SNR is in interval $I_{i}$ over the time interval $[0, n]$. Therefore we can achieve the transmission rate of

$$
R^{(n)}=\sum_{i=0}^{m M} \frac{N_{i} R_{i}}{n}=\sum_{i=0}^{m M} B \log \left(1+\frac{\xi_{i} \gamma_{i}}{\bar{P}}\right) \frac{N_{i}}{n}
$$

Taking the limit of $R^{(n)}$ in (21) as $n \rightarrow \infty$ and using the property (17) we have (steps have been omitted)

$$
\lim _{n \rightarrow \infty} R^{(n)}=\sum_{i=0}^{m M} B \log \left(1+\frac{\xi_{i} \gamma_{i}}{\bar{P}}\right) \int_{\gamma_{i}}^{\gamma_{i+1}} f(\gamma) d \gamma
$$

Thus, for any $\epsilon>0$ there is a sufficiently large $n$ such that

$$
R^{(n)} \geq \sum_{i=0}^{m M} B \log \left(1+\frac{\xi_{i} \gamma_{i}}{\bar{P}}\right) \int_{\gamma_{i}}^{\gamma_{i+1}} f(\gamma) d \gamma-\epsilon .
$$

Clearly, the value of the received SNR for the codeword $w_{i}$ is not less than $\frac{\xi_{i} \gamma_{i}}{\bar{P}}$ for the codes from the $i^{t h}$ sub-codebook, therefore the error probability of the multiplexed coding scheme is bounded by the union bound $\epsilon_{n} \leq \sum_{i=0}^{m M} \epsilon_{n, i}$ where $\epsilon_{n} \rightarrow 0$ as codeword length $n$ becomes infinitely large, because $n \rightarrow \infty$ implies that either $n_{i} \rightarrow \infty$ or there is no power allocated in the interval $I_{i}$. In other words, $I_{i} \notin \mathcal{D}(P)$, which indicates that there is no input for these channel states and thus no encoding or decoding will be performed.

From the assumption of the theorem, $P(\gamma) \in \mathcal{P}$, therefore

$$
R=\int_{\mathcal{D}(P)} B \log \left(1+\frac{P(\gamma) \gamma}{\bar{P}}\right) f(\gamma) d \gamma<\infty
$$

thus, for a given $\epsilon>0$ there exist an (integer) value $M$, such that

$$
\int_{\rho+M}^{\infty} B \log \left(1+\frac{P(\gamma) \gamma}{\bar{P}}\right) f(\gamma) d \gamma<\epsilon
$$

On the other hand, for a fixed value of $M$, the monotone convergence theorem [12] implies that

$$
\begin{aligned}
& \lim _{m \rightarrow \infty} \sum_{i=0}^{m M} B \log \left(1+\frac{\xi_{i} \gamma_{i}}{\bar{P}}\right) \int_{\gamma_{i}}^{\gamma_{i+1}} f(\gamma) d \gamma \\
& =\lim _{m \rightarrow \infty} \sum_{i=0}^{m M} \int_{\gamma_{i}}^{\gamma_{i+1}} B \log \left(1+\frac{\xi_{i} \gamma_{i}}{\bar{P}}\right) f(\gamma) d \gamma \\
& =\int_{\rho}^{\rho+M} B \log \left(1+\frac{P(\gamma) \gamma}{\bar{P}}\right) f(\gamma) d \gamma .
\end{aligned}
$$

where the last equation is obtained by using the definition of $\xi_{i}$ in (16). Thus, for a given $\epsilon>0$ there is a sufficiently large $m$ such that

$$
\begin{aligned}
\sum_{i=0}^{m M} B \log & \left(1+\frac{\xi_{i} \gamma_{i}}{\bar{P}}\right) \int_{\gamma_{i}}^{\gamma_{i+1}} f(\gamma) d \gamma \\
& \geq \int_{\rho}^{\rho+M} B \log \left(1+\frac{P(\gamma) \gamma}{\bar{P}}\right) f(\gamma) d \gamma-\epsilon .
\end{aligned}
$$

By combining the (23), (25), and (27) we conclude that for any $\epsilon>0$ there exist the values of $n, m$, and $M$ sufficiently large such that

$$
R^{(n)} \geq \int_{\rho}^{\infty} B \log \left(1+\frac{P(\gamma) \gamma}{\bar{P}}\right) f(\gamma) d \gamma-3 \epsilon .
$$

Thus by letting all three values of $n, m$, and $M \rightarrow \infty$ any rate close to $R$ in (14) is achievable, and this completes the proof of the coding theorem.

It is worth noting that the condition on the class of power adaption scheme in Theorem 2 is satisfied by all the power adaption policies subject to both peak and average power constraint defined as the class $\mathcal{F}_{1}$ in (11). Therefore, for any power adaption policy in the class $\mathcal{F}_{1}$, there exists a coding scheme which achieves the rate $R$ in (14) with vanishing error probability as codeword length goes to infinity. Specifically, we have the following corollary.

Corollary 1: The class of power adaption policies $\mathcal{F}_{1}$ defined in (11) satisfies the condition of the class $\mathcal{P}$ in Theorem 2, i.e., $\mathcal{F}_{1} \subset \mathcal{P}$.

Proof: Proof simply follows from Jensen's Inequality.

\section{Converse}

In this section we derive a bound on the achievable rate of any coding scheme for which the input power allocation belongs to a given class of power allocation policies, say $\mathcal{P}$. To find this bound we assume that both the transmitter and the receiver have noncausal and perfect knowledge of the sequence of the fading states, or equivalently received SNRs $\left\{\gamma_{i}\right\}_{i=1}^{\infty}$. The proof is based on the newly derived min-cut max-flow theorem for multi-state networks [9], [10]. To be able to use this bound we first partition the range of the received SNRs to finite number of intervals and then take the limit as the number of intervals grows infinitely. 
Theorem 3: The achievable rate, $R$, of any coding scheme for which the input power distribution, $P(\gamma)$, belongs to the class of input power adaption policy $\mathcal{P}$ is bounded above by

$$
R \leq \sup _{P \in \mathcal{P}} \int_{\mathcal{D}(P)} B \log \left(1+\frac{P(\gamma) \gamma}{\bar{P}}\right) f(\gamma) d \gamma
$$

where $\mathcal{D}(P)$ is defined as

$$
\mathcal{D}(P)=\{\gamma: P(\gamma) \neq 0\}
$$

Proof: It has been shown in [9], [10] that for a multi-state single link channel if the sequence of states of the network are non-causally known to both of the transmitter and the receiver, then the achievable rate of information transfer over $n$ uses of the channel is upper-bounded by

$$
R \leq \sum_{m=1}^{M} \frac{N_{m}}{n} I\left(X_{(m)} ; Y_{(m)}\right)
$$

where $M$ is the number of states of the channel, $N_{m}$ denotes the number of times that state $m$ is used in past $n$ uses of the channel, $X_{(m)}$ denotes the input and $Y_{(m)}$ is the output where channel is used in state $m$. Let $M(n)$ denotes the possible states of the network in first $n$ channel uses and for any $m=$ $1,2, \ldots, M(n)$ define $\gamma(m)$ to be the received SNR in state $m$. Since the value of $\gamma(m)$ is unique for different $m$, set of $\{\gamma(m)\}_{m=1}^{M(n)}$ can also be used to denote the set of possible state of the channels. Let $N(n, \gamma)$ denotes the number of times that channel is used in state $\gamma$ and $\mathcal{D}(n, P)$ denote the set of all observed $\gamma(m)$ over the first $n$ channel uses. during first $n$ channel uses. Clearly as $n \rightarrow \infty$ the number of times that channel is in the state $\gamma$ for the first $n$ channel uses approaches to $n f(\gamma) d \gamma$.

Since for any state $\gamma$ the value of the state variable $\gamma$ is known to both of the receiver and transmitter, the channel is equivalent to AWGN channel with average noise power $\frac{\bar{P}}{\gamma}$, and from the power control policy, $P(\gamma)$ denotes the average power used in state $\gamma$. Therefore, the maximum of the mutual information $I\left(X_{(m)} ; Y_{(m)}\right)$ is given by

$$
I\left(X_{(m)} ; Y_{(m)}\right) \leq B \log \left(1+\frac{P(\gamma(m)) \gamma(m)}{\bar{P}}\right)
$$

By combining (31) and (32) and taking the limit as $n$ goes to infinity, we have

$$
\begin{array}{r}
R \leq \lim _{n \rightarrow \infty} \sum_{m=1}^{M(n)} \frac{N_{m}}{n} B \log \left(1+\frac{P(\gamma(m)) \gamma(m)}{\bar{P}}\right) \\
\leq \lim _{n \rightarrow \infty} \int_{\mathcal{D}(n, P)} \frac{N(n, \gamma)}{n} B \log \left(1+\frac{P(\gamma) \gamma}{\bar{P}}\right) \\
\leq \int_{\mathcal{D}(P)} f(\gamma) B \log \left(1+\frac{P(\gamma) \gamma}{\bar{P}}\right) d \gamma
\end{array}
$$

since as $n \rightarrow \infty$ the proportion of times of being in state $\gamma$ approaches the $f(\gamma) d \gamma$, and also the set $\mathcal{D}(n, P) \rightarrow \mathcal{D}(P)$ since each state $\gamma$ with nonzero $f(\gamma)$ would have nonzero probability of occurrence over $n$ independent channel use for sufficiently large $n$.

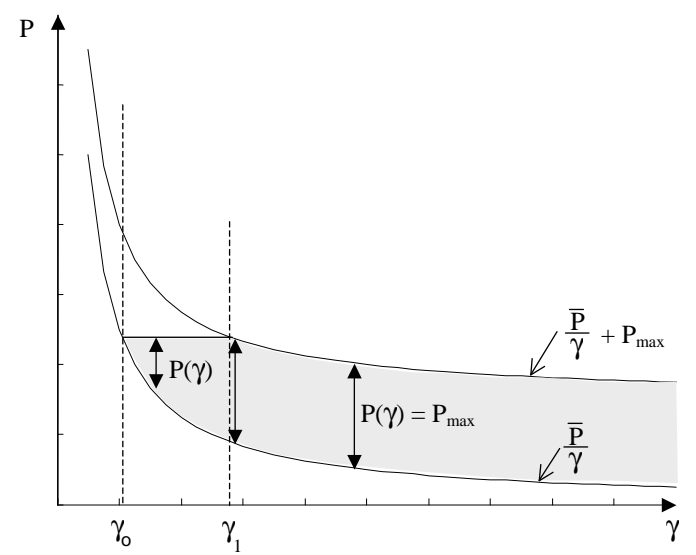

Fig. 2. Optimal power adaption for fading channel with both peak and average power constraint

Therefore, the supremum of the above bound over all power control policies $P \in \mathcal{P}$ defined in (29) establishes an upperbound on the achievable rate of all coding schemes for which its input power belongs to the class of policies $\mathcal{P}$, and this completes the proof.

Now we consider the class $\mathcal{F}_{1}$ of the power control policies defined in (11) and find the power adaption policy $P(\gamma) \in \mathcal{F}_{1}$ which gives the supremum value of the bound of (29). It is not hard to see that the problem

$$
\begin{array}{cl}
\underset{P(\gamma)}{\operatorname{Maximize}} \int_{\mathcal{D}(P)} B \log \left(1+\frac{P(\gamma) \gamma}{\bar{P}}\right) f(\gamma) d \gamma \\
\text { Subject to } & E_{\gamma}[P(\gamma)] \leq \bar{P} \\
& \forall \gamma: P(\gamma) \leq P_{\max }
\end{array}
$$

is a constrained convex optimization problem which can be easily solved by lagrange multiplier techniques [13]. By applying Karush-Kuhn-Tucker (KKT) optimality conditions, the solution of $P(\gamma)$ is obtained which is equivalent to the power adaption policy defined in (8), (9), and (10) in Theorem 1. Furthermore, it is quite easy to verify that this power adaption policy belongs to class $\mathcal{F}_{1}$. Thus, the rate of (7) is achievable for a code that its power distributions belongs to the class $\mathcal{F}_{1}$ through the coding scheme of the Theorem 2. Therefore, we have shown both the achievablity and the converse for the rate in Theorem 1 and also the fact that the power control policy is the solution to the optimization problem defined in (34).

\section{Numerical Results}

In this section, we first demonstrate a visualization of the optimal power adaption for fading channels with both peak and average power constraints. Then, the capacity plots will be presented. It is clear that for a given peak power value, $P_{\max }$, if we relax the average power $\bar{P}$ the capacity would be limited to the capacity with constant power adaption of (3) and will not grow with increasing the input average power beyond the peak power, i.e. $\bar{P} \geq P_{\max }$.

The optimal power adaption defined in (8)-(10) is represented in Figure 2. We recognize three regions which are 


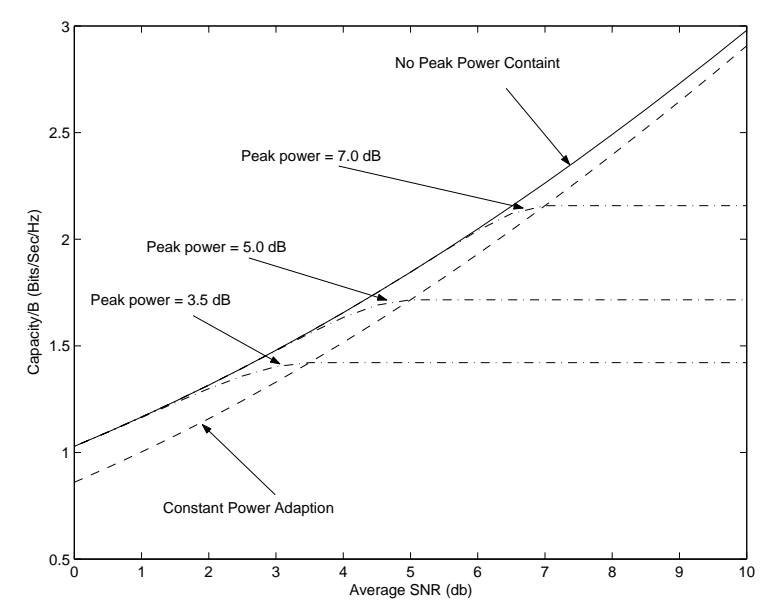

Fig. 3. Capacity of the Rayleigh fading channel with different power constraint

distinguished by two instantaneous received SNR thresholds $\gamma_{0}$ and $\gamma_{1}$. When the received SNR is below $\gamma_{0}$ no transmission occurs, but if the received SNR is greater than $\gamma_{0}$ but less than $\gamma_{1}$ power adaption is in the form of water-filling. Finally, for the values of received SNR greater than $\gamma_{1}$, constant power equal to the peak power constraint is used.

The capacity of the Rayleigh fading channel with both peak and average power versus normalized average input power, defined as $\frac{P}{\mathcal{N}_{0} B}$ is depicted in Figure 3 for the various peak power constraint. The capacity of Rayleigh fading channel without peak power constraint [4] for both case of perfect CSI at the transmitter and receiver $C_{T \& R C S I}$, i.e., water-filling power adaption, and the receiver channel state information only $C_{R C S I}$, i.e. constant power adaption, are also shown as a reference. The horizontal axis, the normalized average input power $\frac{\bar{P}}{\mathcal{N}_{0} B}$, corresponds to average received SNR for the capacity plots of $C_{T \& R C S I}$ and $C_{R C S I}$, so it is labeled accordingly. However, it is in fact the normalized available input average power when we interpret the other capacity plots in this figure.

It can be noted that the capacity with both peak and power constraint is the same as $C_{T \& R C S I}$ for low SNR but as the average input power approaches to the peak power constraint, it becomes flat and hits the curve $C_{R C S I}$ at the $C_{R C S I}\left(P_{\max }\right)$ and remains constant afterward.

It is a well-known fact that the capacity gain becomes "negligible" [4] for high SNR. Therefore, the capacity plots of Figure 3 and Figure 4 are shown over the range of SNRs where the capacity gain from water-filling adaption over constant power adaption is still considerable. We are expecting to lose even more in capacity when the input is more constrained, i.e. both peak and average power constraint exist. Figure 4 shows the capacity for the case that the peak power constraint is in the form of the fixed peak to average power ratio (PAR). Surprisingly, for the range of SNR depicted in Figure 4 the loss in capacity is not considerable even for the PAR $=1.7$. The capacity with PAR $=1.1$ is also depicted in Figure 4 which shows the trend of the capacity in this case. The capacity with constant peak to average power ratio is equal to the capacity

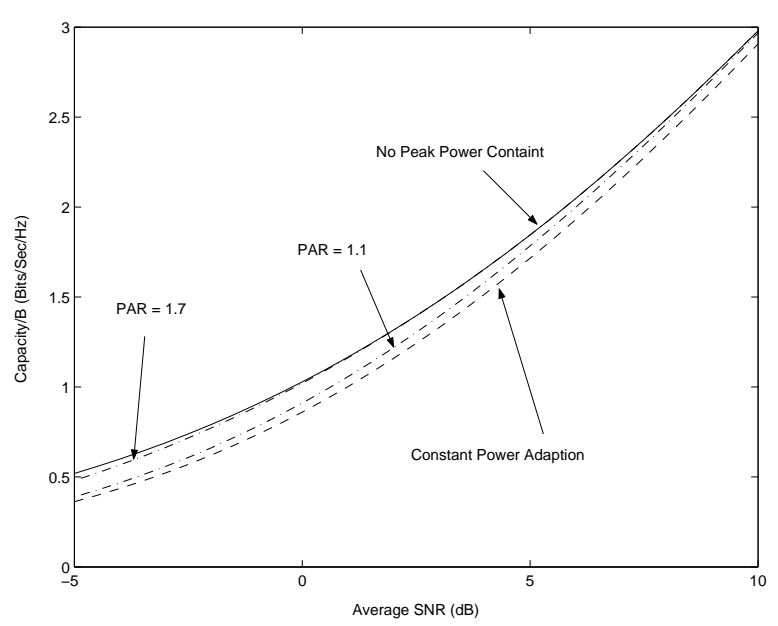

Fig. 4. Capacity of the Rayleigh fading channel for fixed peak to average power ratio

with receiver side information only, $C_{R C S I}$, for low values of SNR and it diverges from this capacity and approaches the capacity $C_{T \& R C S I}$ for higher values of SNR.

\section{CONCLUSION}

We have derived the capacity of fading channel subject to both peak and average power constraint. It has been shown that the capacity is achieved with variable power multiplex gaussian codebook. The optimal power adaption based on the fading state of the channel is divided into three separate regions: (i) zero power allocation (no transmissions) (ii) waterfilling power allocation (iii) constant power allocation.

\section{REFERENCES}

[1] C. E. Shannon, "A mathematical theory of communication," Bell System Technical Jornal, vol. 37.

[2] J. G. Smith, "The information capacity of amplitude and variance constrained scalar gaussian channel," Inform. Contl., vol. 18.

[3] S. Shamai and I. Bar-David, "The capacity of average and peakpower-limited quadrature gaussian channels," IEEE Transactions on Information Theory, vol. 41, no. 4, pp. 1060-1071, 1995.

[4] A. J. Goldsmith and P. Varaiya, "Capacity of fading channels with channel side information," IEEE Transactions on Information Theory.

[5] T. Ericson, "A gaussian channel with slow fading," IEEE Transactions on Information Theory, vol. 16, no. 3, pp. 353 -355, May 1970.

[6] G. Ungerboeck, "Channel coding with multilevel/phase signals," IEEE Transactions on, vol. 28, no. 1, pp. 55-67, Jan. 1982.

[7] G. J. Forney, R. Gallager, G. Lang, F. Longstaff, and S. Qureshi, "Efficient modulation for band-limited channels," IEEE Journal on Selected Areas in Communications, vol. 2, no. 5, pp. 632 -647, Sept. 1984.

[8] E. Biglieri, D. Divsalar, P. J. McLane, and M. K. Simon, Intorduction to Trellis-Coded Modulation with Applications. New York: MacMillan, 1991.

[9] M. A. Khojastepour, A. Sabharwal, and B. Aazhang, "Bounds on achievable rates for general multi-terminal networks with practical constraints," in Lecture Notes in Computer Science, LNCS2634, 2003.

[10] —, "Cut-set theorems for multi-state networks," Submitted for publication in the IEEE Trans. on Information Theory.

[11] R. McEliece and W. Stark, "Channels with block interference," IEEE Transactions on Information Theory, vol. 30, no. 1, pp. 44-53, Jan. 1984.

[12] P. Billingsley, Probability and Measure. 2nd ed., New York: Wiley, 1986.

[13] R. Tapia, Mathematical Optimization and Lagrange Multiplier Theory for Scientists and Engineers. Course notes CAAM-460, Rice University, Houston, TX, 2000. 